\title{
REPORT: AN EXAMINATION OF A DECISION PROCEDURE
}

\author{
BY F. C. OGLESBY
}

\section{Communicated by Everett Pitcher, February 5, 1961}

1. Introduction. R. Stanley in [5] presents a proof procedure for the universal validity (validity in all nonempty domains) of formulas of the first order predicate calculus which is relatively easy to apply. He shows that his procedure is a decision procedure for the monadic predicate calculus. Further, he states that the limits of the procedure, short of being a general method of decision, are not known, but that every universally valid formula which he has tested has been shown by his procedure to be valid.

W. Ackermann, in his review of Stanley's paper [1], gives an example of a universally valid formula of degree higher than two (see $\$ 3$ below) for which Stanley's procedure gives no decision and also suggests a way to enlarge the realm of application of the procedure; namely, to start not with a given formula $A$, but with a finite disjunction $A \vee \cdots \vee A$.

The purpose of this note is to report on an examination of Stanley's procedure to determine for certain decidable classes of formulas whether or not the procedure gives a method of decision. We shall use SP to denote Stanley's procedure.

Throughout this note, the familiar propositional connectives are denoted by ' $\wedge$ ' (and), ' $\vee$ ' (or), and ' $\neg$ ' (not). Individual variables are denoted by $x, x_{1}, x_{2}, \cdots, y, y_{1}, y_{2}, \cdots$, and predicate variables by $F_{1}^{1}, F_{2}^{1}, \cdots, F_{1}^{2}, F_{2}^{2}, \cdots, G_{1}^{2}, G_{2}^{2}, \cdots, H_{1}^{2}, H_{2}^{2}, \cdots$, the superscript indicating the monadic or dyadic character of the variable. We shall feel free to omit subscripts and superscripts whenever no ambiguity will arise. Universal quantifiers are denoted by $(\forall x)$, $\left(\forall x_{1}\right), \cdots,(\forall y),\left(\forall y_{1}\right), \cdots$. We assume throughout that existential quantifiers and signs of material implication or equivalence have been replaced by their respective usual equivalents. $A, A_{1}, \cdots, B$, $B_{1}, \ldots$ denote arbitrary formulas; $A(x), \cdots, B(x), \cdots$ denote arbitrary formulas with free $x$; etc.

The general method of SP is to derive a contradiction from the negation of the given formula. In particular, a preliminary step of exportation (as characterized in W. Quine [6]) is applied to the universal closure of the given formula, followed by a preliminary step of prefixing a ' $\neg$ '. The remainder of the procedure, which we will de- 
note by SP*, involves cycles of prescribed steps including instantiations of quantifications according to natural deduction techniques and applications of propositional rules. Contradictory disjuncts are dropped as they are uncovered and, if at some step everything vanishes, then the original formula is established as universally valid, or, equivalently, the formula resulting from the preliminary steps is established as nonsatisfiable (satisfiable in no nonempty domain). In this case, we say that SP yields a contradiction.

2. We say that a formula $A$ is a member of the $\mathrm{AE}$ predicate calculus if the formula resulting from $A$ by applying the preliminary steps of SP contains no negative quantifier within the scope of a positive quantifier. (Positive and negative quantifiers are defined as with J. Herbrand [2]; when converted to prenex normal form, positive quantifiers appear in the prefix as universal quantifiers and negative as existential.) Note that by this definition the monadic predicate calculus is contained in the AE predicate calculus. Thus, the following theorem is an extension of Stanley's result that the procedure gives a method of decision for the monadic predicate calculus.

THEOREM 1. SP gives a method of decision for the AE predicate calculus.

Proof. Let $A$ be an arbitrary universally valid formula of the AE predicate calculus. Then $A^{*}$, the result of applying the preliminary steps of SP to $A$, is nonsatisfiable and has an equivalent prenex normal form:

$$
\begin{aligned}
& \neg\left(\forall x_{1}\right) \neg \cdots \neg\left(\forall x_{n}\right) \neg\left(\forall y_{1}\right) \cdots\left(\forall y_{m}\right) B\left(x_{1}, \cdots, x_{n}, y_{1}, \cdots y_{m}\right), \\
& n \geqq 0 \text {. }
\end{aligned}
$$

Hence, as is well-known, $A^{*}$ is not satisfiable in any domain containing $n$ individuals if $n>0$, and $A^{*}$ is not satisfiable in any domain containing one individual if $n=0$. Thus, upon applying to $A^{*}$ the procedure in Hilbert and Ackermann [3] for determining satisfiability in finite domains, a truth-functional contradiction is obtained. It follows easily from this that $\mathrm{SP}^{*}$ applied to $A^{*}$ yields a contradiction.

3. A formula is of second degree if at least one quantifier appears within the scope of another quantifier, but no quantifier appears within the scope of more than one other quantifier. K. J. Hintikka in [4] extends the notion of distributive normal form in the propositional calculus to the full predicate calculus. In particular, he defines closed second degree distributive normal forms. These latter normal forms are finite disjunctions of formulas called closed second degree 
constituents of zero order. Following Hintikka, we denote an arbitrary closed second degree constituent of zero order by $C^{0} 2$. Hintikka presents a set of three conditions with the property that an arbitrary $\mathrm{C}^{0} 2$ is nonsatisfiable if and only if at least one of the conditions holds for $C^{0} 2$. However, a proof of the necessity of these conditions is omitted in his monograph. We obtain a proof of the necessity of these conditions by showing that if none of the conditions holds for an arbitrary $C^{0} 2$, then $C^{0} 2$ is satisfiable in a denumerably infinite domain.

4. TheOREM 2. SP* gives a method of decision for nonsatisfiability in the class of all closed second degree distributive normal forms.

Proof. The theorem follows by showing that SP* yields a contradiction when applied to an arbitrary $C^{0} 2$ satisfying at least one of the above three conditions. It should be mentioned that there are certain special cases for which the set of necessary and sufficient conditions does not apply, but that these cases are easily handled.

5. We say that a formula $A$ of the predicate calculus is a member of the class $\Phi$ if the formula $A^{*}$ which results from applying the preliminary steps of SP to $A$ is (closed and) of second degree. Note that if $A^{*}$ is of first degree (no quantifier appears within the scope of another quantifier), then $A$ is a member of the $\mathrm{AE}$ predicate calculus and Theorem 1 applies.

Since any arbitrary closed second degree formula $A^{*}$ can be effectively transformed into an equivalent closed second degree distributive normal form, the conditions of $\$ 3$ and Theorem 2 each give a method of decision for determining universal validity in the class $\Phi$. However, it is not, in general, practical to attempt the transformation, and hence it is of interest to determine whether SP necessarily yields a contradiction when applied directly to an arbitrary universally valid formula of $\Phi$. The following theorem gives a negative answer to this question.

TheoRem 3. SP is not a decision procedure for determining universal validity of arbitrary formulas of $\Phi$.

Proof. Let $A(x)$ denote

$$
[F x \wedge \neg(\forall y) \neg(G x y \wedge \neg F y)] \vee[F x \wedge \neg(\forall y) \neg(H x y \wedge \neg F y)]
$$

Consider $B_{1}=\neg(\forall x) A(x)$. The universal validity of $B_{1}$ is easily determined by a direct valuation. However, it can be shown that SP applied to $B_{1}$ does not yield a contradiction.

REMARK. We note that it can be shown that SP does yield a contradiction when applied to the formula 
$\neg(\forall x)\{F x \wedge[\neg(\forall y) \neg(G x y \wedge \neg F y) \vee \neg(\forall y) \neg(H x y \wedge \neg F y)]\}$,

which is equivalent to $B_{1}$. However, a simple application of the distributive law does not, in general, remedy the situation; for the following formula, $B_{2}$, is universally valid but does not yield a contradiction under application of SP:

$$
\begin{aligned}
\neg(\forall x)\{[\neg(\forall y) & \neg(F x y \wedge \neg F y y) \wedge F x x] \vee[\neg(\forall y) \neg(F y x \wedge \neg F y y) \\
& \wedge(\forall y)((\neg F x y \wedge \neg F y y) \vee(F y x \wedge F y y)) \wedge \neg F x x]\} .
\end{aligned}
$$

6. Ackermann's suggestion to start not with a given formula $A$, but rather with a finite disjunction $A \vee \cdots \vee A$ does strengthen SP for the class $\Phi$, for it can be shown that SP applied to $B_{1} \bigvee B_{1}$ yields a contradiction. Moreover, it can be shown that although SP applied to $B_{2} \bigvee B_{2}$ does not yield a contradiction, SP applied to $B_{2} \bigvee B_{2} \bigvee B_{2}$ does yield a contradiction (these results also establishing the universal validity of $B_{1}$ and $B_{2}$ ).

It is clear, however, that the suggestion cannot give us a method of decision for the class $\Phi$ unless we can give some sort of a rule for determining the number of disjuncts to be used. For convenience, let us denote SP modified by starting with $q$ disjuncts of the given formula by $\mathrm{SP}_{q}$.

TheOREM 4. No positive integer $M$ exists such that $S P_{M}$ is a decision procedure for determining universal validity of arbitrary formulas of $\Phi$.

Proof. Let $m$ be an arbitrary positive integer. Consider the formula

$$
B_{m}^{\prime}=\neg(\forall x) A_{1}(x) \wedge \neg(\forall x) A_{2}(x) \wedge \cdots \wedge \neg(\forall x) A_{m}(x),
$$

where

$$
\begin{aligned}
A_{k}(x) & =\left[F_{k} x \wedge \neg(\forall y) \neg\left(G_{k} x y \wedge \neg F_{k} y\right)\right] \\
& \vee\left[F_{k} x \wedge \neg(\forall y) \neg\left(H_{k} x y \wedge \neg F_{k} y\right)\right], \quad 1 \leqq k \leqq m .
\end{aligned}
$$

The universal validity of $B_{m}^{\prime}$ is easily established by a direct valuation. However, it can be shown that, if $q \leqq m$, then $\mathrm{SP}_{q}$ applied to $B_{m}^{\prime}$ does not yield a contradiction, whereas $\mathrm{SP}_{m+1}$ applied to $B_{m}^{\prime}$ does yield a contradiction (also establishing the universal validity of $B_{m}^{\prime}$ ).

REMARK. Theorem 4 does not exclude the possibility of being able to give, for the class $\Phi$, an effective rule which, when applied to a given individual formula $A$ of $\Phi$, will produce an integer $N$ (depending upon $A$ ) such that: if $A$ is universally valid, then $\mathrm{SP}_{N}$ applied to $A$ yields a contradiction. 


\section{REFERENCES}

1. W. Ackermann, A review of [5], J. Symb. Logic vol. 21 (1956) p. 197.

2. J. Herbrand, Recherches sur la théorie de la démonstration, Trav. Soc. Sci. Lett. Varsovie, Classe III, no. 33, 1930.

3. D. Hilbert and W. Ackermann, Principles of mathematical logic, New York, Chelsea Publishing Company, 1950.

4. K. J. Hintikka, Distributive normal forms in the calculus of predicates, Acta Philosophica Fennica vol. 6 (1953) 71 pp.

5. R. Stanley, An extended procedure in quantificational logic, J. Symb. Logic vol. 18 (1953) pp. 97-104.

6. W. V. Quine, On the logic of quantification, J. Symb. Logic vol. 10 (1945) pp. 1-12.

\section{LEHIGH UNIVERSITY}

